\title{
Commentary
}

\section{Recent Advancements in Hematology: Knowledge, Methods and Dissemination, Part 2}

\author{
Jill Corre 1,2 ${ }^{1}$, Michele Sabbah ${ }^{3}\left(\mathbb{D}\right.$, Fredrik Schjesvold 4,5 ${ }^{(D}$, Amer M. Zeidan ${ }^{6}$, Francesco Buccisano ${ }^{7,8} \mathbb{D}$, \\ David Sallman ${ }^{9}$, Mario Mazzucato ${ }^{10} \mathbb{D}$, Leigh A. Madden ${ }^{11} \mathbb{D}$, Maurizio Martini ${ }^{12} \mathbb{D}$, Eric Van Breda ${ }^{13} \mathbb{D}$, \\ Riccardo Dolcetti ${ }^{14,15}{ }^{(D)}$, Alessandro Busca ${ }^{16}\left(\mathbb{D}\right.$, Gordon Cook ${ }^{17}$, Francesco Onida ${ }^{18}$ (D), Annibale Versari ${ }^{19}$ (D), \\ Jean-Jacques Kiladjian 20 (D), Roland B. Walter ${ }^{21, *(\mathbb{D}) \text {, Laurent Garderet }}{ }^{22, *}$, Marie Robin ${ }^{23, *}$ and \\ Alberto Signore $24,25, *$ D
}

check for updates

Citation: Corre, J.; Sabbah, M.; Schjesvold, F.; Zeidan, A.M.; Buccisano, F.; Sallman, D.;

Mazzucato, M.; Madden, L.A.; Martini, M.; Van Breda, E.; et al. Recent Advancements in Hematology: Knowledge, Methods and Dissemination, Part 2. Hemato 2021, 2, 79-88. https://doi.org/ 10.3390/hemato2010004

Academic Editor: Antonino Carbone Received: 12 January 2021 Accepted: 5 February 2021 Published: 9 February 2021

Publisher's Note: MDPI stays neutral with regard to jurisdictional claims in published maps and institutional affiliations.

Copyright: (c) 2021 by the authors. Licensee MDPI, Basel, Switzerland. This article is an open access article distributed under the terms and conditions of the Creative Commons Attribution (CC BY) license (https:// creativecommons.org/licenses/by/ $4.0 /)$.
1 Centre de Recherche en Cancérologie de Toulouse, Institut National de la Santé et de la Recherche Médicale U1037, F-31059 Toulouse, France; corre.jill@iuct-oncopole.fr

2 Unit for Genomics in Myeloma, Institut Universitaire du Cancer de Toulouse-Oncopole, University Hospital, F-31059 Toulouse, France

3 Centre de Recherche Saint-Antoine, INSERM U938, Sorbonne Université, F-75012 Paris, France; michele.sabbah@inserm.fr

4 Oslo Myeloma Center, Oslo University Hospital, 0424 Oslo, Norway; fredrikschjesvold@gmail.com

5 KG Jebsen Center for B Cell Malignancies, University of Oslo, 0424 Oslo, Norway

6 Section of Hematology, Department of Internal Medicine, Yale University School of Medicine, New Haven, CT 06520-8028, USA; amer.zeidan@yale.edu

7 Hematology, Department of Biomedicine and Prevention, University Tor Vergata, 00133 Rome, Italy; francesco.buccisano@uniroma2.it

8 Fondazione Policlinico Tor Vergata, 00133 Rome, Italy

9 Department of Hematology and Oncology, H. Lee Moffitt Cancer Center and Research Institute, Tampa, FL 33612, USA; David.Sallman@moffitt.org

10 Centro di Riferimento Oncologico di Aviano (CRO) IRCCS Aviano, via F. Gallini n. 2, 33081 Aviano, Italy; mmazzucato@cro.it

11 Department of Biomedical Science, The University of Hull, Hull HU6 7RX, UK; 1.a.madden@hull.ac.uk

12 Institute of Pathology, Catholic University of Sacred Heart, Largo A. Gemelli 8, 00168 Rome, Italy; maurizio.martini@unicatt.it

13 Department of Rehabilitation Sciences \& Physiotherapy, Antwerp Multidisciplinary Metabolic Research Unit (AM2RUN), University of Antwerp, B-2000 Antwerp, Belgium; eric.vanbreda@uantwerpen.be

14 Sir Peter MacCallum Centre for Cancer Immunotherapy, University of Melbourne, Parkville 3000, Australia; r.dolcetti@uq.edu.au

15 Diamantina Institute, Faculty of Medicine, The University of Queensland, Brisbane 4102, Australia

16 Stem Cell Transplant Center, Azienda Ospedaliera, Universitaria Città della Salute e della Scienza di Torino, 10126 Turin, Italy; abusca@cittadellasalute.to.it

17 Section of Experimental Haematology, Leeds Institute of Cancer \& Pathology, University of Leeds, St James's University Hospital, Beckett Street, Leeds LS9 7TF, UK; G.Cook@Leeds.ac.uk

18 Fondazione IRCCS Ca' Granda Ospedale Maggiore Policlinico, University of Milan, 20122 Milan, Italy; francesco.onida@unimi.it

19 Nuclear Medicine Unit, Azienda USL-IRCCS di Reggio Emilia, Viale Risorgimento, 8042123 Reggio Emilia, Italy; annibale.versari@ausl.re.it

20 Centre d'Investigations Cliniques, APHP, Hôpital Saint-Louis, Université de Paris, INSERM CIC, 1427, F-75000 Paris, France; jean-jacques.kiladjian@aphp.fr

21 Clinical Research Division, Fred Hutchinson Cancer Research Center, Seattle, WA 98109, USA

22 Service d'Hématologie, Hôpital Pitié Salpêtrière, 47-83 boulevard de 1' hôpital, F-75013 Paris, France

23 Hôpital Saint-Louis, Université de Paris, APHP, INSERM 1131, 75000 Paris, France

24 Nuclear Medicine Unit, Department of Medical-Surgical Sciences and of Translational Medicine, "Sapienza" University of Rome, 00161 Rome, Italy

25 Department of Nuclear Medicine and Molecular Imaging, University of Groningen, 9700 Groningen, The Netherlands

* Correspondence: rwalter@fredhutch.org (R.B.W.); laurent.garderet@aphp.fr (L.G.); marie.robin@aphp.fr (M.R.); alberto.signore@uniroma1.it (A.S.) 


\section{Introduction}

Recent Advancements in Hematology: Knowledge, Methods and Dissemination is a series of commentary article which is published on a biannual basis by the editorial board of the journal Hemato. In these articles, we highlight in brief reports (of about one hundred words) a number of recently published articles that describe the most recent advancements in hematology. In this way, we hope Hemato will not only publish high-level scientific articles, but will also discuss their significance in the current scientific scenario, which includes the transfer of information to the media.

\section{Prognostic Mutations in Multiple Myeloma Highlighted by Jill Corre}

Despite major outcome improvement in multiple myeloma, some patients still experience a very poor outcome. Furthermore, some early relapse without any obvious reason since classical high-risk cytogenetic factors are absent, suggesting that other factors need to be discovered. With the advent of next generation sequencing, we learnt that multiple myeloma reveals many mutations but very few are recurrent, preventing so far the establishment of their prognostic impact.

However, this was without counting on the work of Boyle et al. [1]. By sequencing the tumor DNA of 223 newly diagnosed patients included in the Total Therapy trials, they were able to demonstrate that, in addition to previously described biallelic inactivation of TP53 [2], DIS3 and BRAF mutations are also associated with poor outcome. The key to this success lies in a remarkably long clinical follow-up (more than eight years) and the use of a sequencing panel targeting 125 mutations of interest. Interestingly, amongst the non-V600E $B R A F$ mutations, the $44 \%$ identified as hypoactive/dead kinase showed co-occurrence with mutations in KRAS or NRAS, probably facilitating MAPK inactivation.

\section{Bone Marrow Adipocyte Reprogramming by Multiple Myeloma Cells Highlighted by Michèle Sabbah}

The pathogenesis of multiple myeloma (MM) involves complex, bidirectional interactions of MM cells with bone marrow resident cells including bone marrow adipocytes (BMA). Some studies have demonstrated that BMA are reprogrammed by MM and contribute to myeloma-induced bone disease [3]. Fairfield et al. studied the cross-talk between BMA and MM cells. Using patient-derived samples and murine models, the authors provide evidence that adipocytes exposed to myeloma-derived factors exhibited decreased lipid content, decreased adipogenic transcripts and increased expression of senescenceassociated secretory phenotype (SASP) transcripts and proteins [4]. Particularly, they observed increases in IL6 and IL8 gene expression and secreted protein in adipocytes upon MM co-culture. These modified adipocytes, in turn, increased the mitotic activity of MM cells and provided resistance to dexamethasone-induced cell cycle arrest. This report highlighted the fundamental part of the microenvironmental cells in myeloma progression, opening the way to new therapeutic approaches such as senolytic therapies for diminished bone damage and myeloma drug resistance.

\section{BELLINI-A Trial with Many Lessons \\ Highlighted by Fredrik Schjesvold and Gordon Cook}

The efficacy of venetoclax has been established for some time in patients with translocation 11;14, and in patients with high BCL2 expression. In 2017, Moreau et al. published results from the combination of bortezomib-dex with venetoclax and showed high response rates [5]. However, most responding patients were bortezomib non-refractory, and of the eight responding bortezomib-refractory patients, three where $t(11 ; 14)$ positive, and an unknown number were high BCL2-expressors. The BELLINI trial challenged bortezomib-dex with the addition of venetoclax in a randomized phase-3 trial [6], but the interim analysis showed an increased mortality in the intervention arm caused by infections, inducing a temporary halt in venetoclax studies globally. Strikingly, there was a clear benefit in terms 
of progression-free survival (PFS) for the venetoclax arm, providing a clear separation between PFS and OS results. Looking at sub-groups it became clear that the increased PFS was seen in patients with $\mathrm{t}(11 ; 14)$ and/or high BLC2-expression, while the increased mortality was seen in patients without these. In conclusion, the trial confirmed a biomarker-driven approach, and reminded us that PFS is not always a surrogate for overall survival.

\section{The Nobel Prize Awarded CRISPR/CAS9 Technology to Cure $\beta$ Thalassemia and Sickle Cell Disease?}

Highlighted by Laurent Garderet

Transfusion-dependent $\beta$ thalassemia (TDT) and sickle cell disease (SCD) are the most common monogenic diseases worldwide. One treatment is to switch hemoglobin production towards fetal hemoglobin $(\mathrm{HbF})$ which is prevented by a transcription factor named BCL11A.The authors first demonstrated that using the CRISPR/CAS9 technology in healthy donors' CD34+ stem cells, they were able to suppress the gene BCL11A and therefore to switch the hemoglobin production mostly in $\mathrm{HbF}$. In two patients, one TDT and one SCD, they collected peripheral CD34+ stem cells, genetically modified the hemoglobin production, and proceeded to an autologous CD34+ peripheral stem cell transplantation after a busulfan myeloablative dose regimen. They demonstrated that, one year after the transplantation, the patients produced a majority of $\mathrm{HbF}$ and became transfusion independent. They, however, experienced transplant-related complications such as aplasia, infections and veno-occlusive liver disease in the TDT patient [7]. The updated results in eight additional patients with a longer follow-up are very encouraging [8]. This is the first proof of principle of a potential cure for TDT and SCD. Its widespread use remains to be determined.

\section{Venetoclax for Acute Myeloid Leukemia-An Ultimate Game Changer? Highlighted by Amer M. Zeidan}

Most patients with acute myeloid leukemia (AML) are older than 65 years of age at time of diagnosis and are ineligible for intensive curative-intent approaches. Older/unfit patients have traditionally been treated with palliative-intent hypomethylating agents (HMA). Early phase trials of the oral BCL-2 inhibitor venetoclax in combination with HMA led to high rates of durable complete response (CR) or CR with incomplete count recovery (CRi) leading to accelerated approval in the USA in November 2018 [9]. The VIALE-A trial, published in NEJM on August 2020, was a randomized phase 3 trial of 431 older/unfit patients with newly diagnosed AML [10]. Patients in the azacitidine-venetoclax group compared to the azacitidine-placebo group had improved overall survival (median 14.7 vs. 9.6 months, $p<0.001)$, and higher CR/CRi rates $(66 \%$ vs. $28 \% ; p<0.001)$, solidifying this regimen as the standard of care. Ongoing trials are adding novel agents to the HMA-venetoclax backbone with the hope that triplets can further improve clinical outcomes. Venetoclax is also being combined with various intensive chemotherapies and with novel and targeted agents such as IDH or FLT3 inhibitors as well as oral HMAs. This could open the door for total oral and chemotherapy-free regimens for non-acute promyelocytic leukemia AML, something that could not have been imagined a few years ago.

\section{7. "The Right Thing at the Wrong Time Is the Wrong Thing" (cit. J. Harris) in AML Highlighted by Francesco Buccisano}

Acute Myeloid Leukemia (AML) is generally considered a medical emergency deserving immediate treatment. Rollig et al. [11] challenged this assumption in a large series of 2263 real-life AML patients treated according to intensive German Study Alliance (SAL)-AML protocols. Difference in time from diagnosis to treatment start (TDT) of 0 to 5 , 6 to 10,11 to 15 or $>15$ days did not show any impact in two-year Overall Survival (OS) neither in univariable nor in multivariable analysis. Furthermore, TDT did not modify prognosis even after stratification for age and leukocyte count. However, patients with a leukocyte count higher than $50,000\left(\times 10^{9} / \mathrm{L}\right)$ were more commonly represented in the groups with shorter TDT suggesting that hyperleukocytosis was timely managed. The 
key message of the study is that, although AML deserves urgent treatment in the majority of cases, in many patients an operational time interval is allowed. In the current era of personalized medicine, this time may be properly used to achieve a complete biological profile or to address comorbidities that could otherwise jeopardize the efficacy of treatment.

\section{Clonal Burden of TP53 Predicts Outcomes in Acute Myeloid Leukemia Patients Highlighted by David Sallman}

Mutation of TP53 imparts a profound negative impact on overall survival (OS) in patients with myelodysplastic syndromes (MDS) and acute myeloid leukemia (AML). In MDS, recent data have identified that the variant allele frequency (VAF) of TP53 further stratifies prognosis over binary mutation analysis alone [12,13]. Short et al. have recently published on the impact of TP53 VAF and outcomes for AML patients undergoing frontline therapy [14]. Notably, a VAF $>40 \%$ was an independent predictor for an inferior relapse free survival and OS, particularly for patients receiving cytarabine-based chemotherapy (median OS 4.7 months). However, patients without a biallelic mutation and with VAF $<40 \%$ did have better outcomes to cytarabine based therapy (median OS 17.6 months), although this is a rare subset of patients (14\% of cohort). Unfortunately, the addition of venetoclax to hypomethylating agent therapy had no improvement on OS, irrespective of baseline TP53 VAF (median OS 4-7 months across cohorts). Together, these data further support the incorporation of TP53 VAF in the prognostication of MDS/AML patients and highlight the profound clinical need for novel therapeutic strategies in this molecular cohort.

\section{First Line Autologous Versus Allogeneic Transplantation in ALK Negative Peripheral T Lymphoma Highlighted by Marie Robin}

Schmitz et al. [15] report the final result of the randomized phase 3 trial comparing autologous (auto) vs allogeneic (allo) transplantation as part of first line therapy in poor risk peripheral T-lymphoma. This multicentric trial was conducted in France and Germany and was pre-published online in Blood Journal. Patients were randomized upfront and received transplantation if they had obtained stable disease or better. One hundred and four patients with peripheral T lymphoma, excluding ALK+ anaplastic lymphoma, were included and 103 patients were analyzed: 44 assigned to auto and 49 to allo. Thirty-four (63\%) from auto arms and 26 (53\%) from allo arm received the transplantation; this low proportion of transplanted patients was related mainly to disease progression. Eight patients from allo arm received auto. By intent-to-treat, outcome was similar in auto and allo arm. Relapse risk was higher with auto while non-relapse mortality was higher with allo. In patients undergoing the transplantation, the three-year overall survival was $57 \%$ after allo and $70 \%$ after auto, and event-free survival at three years was $43 \%$ after allo and $38 \%$ after auto. Although a relatively small proportion of randomized patients received transplantation, this trial shows that standard therapy followed by high dose therapy and autologous transplantation is a valid option in younger patients with peripheral T-cell lymphoma. Due to higher non-relapse mortality after allogeneic transplantation, the authors suggested that this may be proposed in patients with high risk of progression or relapse.

\section{SARS-COV-2: Crosstalk among Inflammation, Immunity and Hemostasis Highlighted by Mario Mazzucato}

Many biochemical and cellular pathways links hemostasis and the innate immune system. Inside this mechanism the endothelial cell maintains hemostatic balance by variably expressing anticoagulant and procoagulant molecules.

Von Willebrand Factors (VWF) is a multimeric protein that is released into the circulation from endothelial stores in a highly thrombogenic form, characterized by the presence of ultra-large multimers. VWF is also a marker of endothelium activation, being massively released after inflammation-mediated vascular damage [16]. 
VWF carries coagulation factor VIII and mediates platelet-vessel wall and platelet-toplatelet interaction, especially at high shear. Under normal circumstances, these ultra-large multimers are cleaved by the protease ADAMTS-13 and, consequently, the high thrombogenicity of released VWF is reduced. The imbalance between high molecular weight VWF multimers and ADAMTS-13 could cause a prothrombotic state in inflammatoryinduced conditions, as observed in sepsis associated with disseminated intravascular coagulation. Recently, huge VWF plasma levels associated with a low ADAMTS-13 activity were observed in patients with severe COVID-19. The VWF-ADAMTS13 imbalance further increases the hypercoagulable state promoted by COVID-19 disease and the risk of micro-thrombosis in these patients [17].

The major blood proteolytic cascades, the complement-coagulation system, have common evolutionary origins to fight infections and prevent exsanguination from wounds [18]. Crosstalk between these systems is thought to have evolved to increase host resilience, but dysregulated interplay between coagulation and immune system may represent a serious health problem. In this context, the COVID-19 pandemic is creating a new paradigmatic example in the nexus between immunological response against SARS-COV-2 virus and the coagulation system.

Ramlall [19] et al. identified, in a retrospective observational study, putative transcriptional genetic markers involved in complement and coagulation-associated dysregulation. Patients with complement dysfunction (activation) and coagulation disorders were at significantly increased risk of mechanical respiration and death following SARS-CoV-2 infection. Conversely, no patients with complement deficiency, associated with increased risk of infections, required mechanical respiration or succumbed to their illness. The data highlight the relevance of therapeutic strategies involving the complement-coagulation mechanism in SARS-COV-2 disorder. Based on these observations, the use of clinically approved anti-C5 mAb eculizumab showed that complement inhibition affords significant therapeutic benefit in COVID-19 patients by intercepting key SARS-CoV-2-induced thrombo-inflammatory pathways. This robust anti-inflammatory response, induced by eculizumab, culminates in respiratory improvement and resolution of COVID19-associated acute respiratory distress syndrome [20].

11. Magnetism and Microfluidics: Isolation and Analysis of Exosomes from Whole Blood Highlighted by Leigh Madden

Exosomes are nanosized extracellular vesicles released into the circulation and are of particular interest in cancer. As noted within the highlighted publication, the detection of these vesicles within blood could be advantageous over, for example, circulating tumor cells or serum biomarkers in cancers. Sancho-Albero et al. [21] used magnetic nanoparticles to which they attached a CD9 antibody to specifically target exosomes, then proceeded to mix these with whole blood taken from pancreatic cancer (PC) patients prior to isolation of exosomes on a microfluidic device with a magnetic field applied. These exosomes were then analysed for CA19-9 protein by ELISA. They showed that CA19-9 levels in exosomes from four PC patients were significantly higher (approximately three-fold) than healthy controls and importantly identified one patient whose low serum CA19-9 concentration would be interpreted as negative for PC. This technique appears to have potential for further studies of exosomes and could also enhance the monitoring of PC patients for recurrence using CA19-9 (for example, Azizian et al. [22]).

\section{Immune-PET Imaging Strategy in Multiple Myeloma}

Highlighted by Alberto Signore

In the era of "personalized medicine", the use of radio-labeled monoclonal antibodies (MoAbs) directed against a therapeutic target was pioneered for selecting patient candidates for immunotherapy. The first radio-labeled MoAbs were anti-CD3, anti-TNFa, and antiCD20 in rheumatic diseases. Then this approach expanded to oncology (anti-HER2, antiPD1, anti-VEGF and others) [23]. Here, we see the use of 64-Copper $\left({ }^{64} \mathrm{Cu}\right)$-anti-CD38 
in hematology for diagnosis of Multiple Myeloma (MM) [24]. What are the relevant novel factors? First is the application of radio-labeled MoAbs in MM as compared with 18F-Fluorodeoxyglucose $\left(\left[{ }^{18} \mathrm{~F}\right] \mathrm{FDG}\right)$, then the use of ${ }^{64} \mathrm{Cu}$ as isotope for positron emission tomography (PET) imaging. ${ }^{64} \mathrm{Cu}$ compared with other PET isotopes, such as $89-Z$ yrconium $\left({ }^{89} \mathrm{Zr}\right)$ which is most frequently used, offers several advantages: its shorter half-life $(12.7 \mathrm{~h})$, being more suitable for imaging, allowing the administration of higher activity, increasing exam sensitivity and limiting the radiation dose to patients. Furthermore, ${ }^{64} \mathrm{Cu}$ shows slow tendency to non-specifically accumulate in bones, which are commonly affected in MM patients and represent a source of possible pitfalls for $\left[{ }^{18} \mathrm{~F}\right] \mathrm{FDG}$. In this first-in-human trial, the authors demonstrate that this approach is safe and feasible in detecting or excluding $\mathrm{MM}$ lesions, and suggest that it could be used instead of $\left[{ }^{18} \mathrm{~F}\right] \mathrm{FDG}$, although larger studies are needed to confirm these initial results.

13. The Rapid Clinical Response to Anti-PD1 in Classic Hodgkin Lymphoma Is Not Mediated by Cytotoxic T-Cell Activation, but a Withdrawal of Pro-Survival Factors in the Tumor Microenvironment

Highlighted by Maurizio Martini

Sarah Reinke et al. [25] have recently contributed to explain the mechanism of antiPD1 efficacy in Classic Hodgkin lymphoma (cHL). Although anti-PD1 effects appear to be largely mediated by cytotoxic CD8+ T cells in solid tumors, scarce Hodgkin and Reed-Sternberg cells (HRSCs) frequently lack major histocompatibility complex and the mechanism of anti-PD1 efficacy is unclear. The authors analyzed paired biopsies and blood samples obtained from a relatively small group of early-stage unfavorable cHL patients that showed rapid clinical responses and high interim complete response rates to anti-PD1 based first-line treatment. The authors observed that HRSCs had disappeared from the tissue within days after the first nivolumab application and also observed a reduction in type 1 regulatory T cells and PD-L1+ tumor-associated macrophages in the tumor microenvironment (TME). Interestingly, a cytotoxic immune response and a clonal T-cell expansion were not observed in the tumors or peripheral blood. In addition, these early changes in the TME were distinct from alterations found in a separate set of cHL biopsies at relapse during anti-PD1 therapy. These data identify a unique very early histologic response pattern to anti-PD1 therapy in cHL that is suggestive, rather than the induction of an adaptive antitumor immune response, as the main mechanism of action.

\section{Unus pro Omnibus, Omnes pro Uno/One for All, All for One, Loyal through Thick and Thin}

Highlighted by Eric van Breda

Laboratory test results which are reported as numbers are not meaningful by themselves unless compared to gold standard reference values. These reference values, also known as 'normal' values, are the loyal friend of health care workers through thick and thin and determine whether a patient is 'healthy' or not. Once values are outside expected ranges the search to identify a possible condition or disease commences.

The rapid onset and worldwide spread of the COVID-19 pandemic (caused by SARS-CoV-2 coronavirus) has been associated with a profound impact on clinical practice. Recently, Mertoglu et al. [26] suggested that routine laboratory tests must change during the COVID-19 pandemic, an interesting phenomenon that sheds a different light on routine laboratory tests as being far more important than regularly thought. If specific tests like the rRT-PCR frequently report negative outcomes even in cases in which patients were indeed infected with the SARS-CoV-2 virus, routine laboratory tests should be regarded as important as specific tests. Especially, hematological parameters like lymphocyte count, d-NLR (derived neutrophil to lymphocyte ratio), LMR (lymphocyte to monocyte ratio) and NLR (neutrophil to lymphocyte ratio) are among hematological markers which can make the difference in the treatment of COVID-19 patients [27,28]. 
Although differences are most likely to be present in the 'reference' values of different laboratories in different countries, it is important for clinical workers to realize that routine tests can be a loyal companion in the fight to predict the outcome of severe COVID-19 cases.

15. A Leukemia-Initiating Fusion Protein Provides a Highly Immunogenic Neoantigen for Improved Immunotherapy of Acute Myeloid Leukemia

Highlighted by Riccardo Dolcetti

Optimal neoantigens for immunotherapy should have critical qualitative features to elicit clinically relevant immune responses, including clonality, marked diversity from selfantigens, similarity to microbial antigens, high protein expression, binding to HLA, and low likelihood of being silenced or deleted [29]. Biernacki et al. [30] have recently demonstrated that the leukemia-initiating $C B F B-M Y H 11$ fusion provides a neoantigen showing all these features. In particular, they have identified a nonameric $\mathrm{CD} 8^{+} \mathrm{T}$-cell epitope derived from the prevalent CBFB-MYH11 fusion protein that is naturally processed and presented on HLA-B * 40:01 by malignant blasts in acute myeloid leukemia (AML) patients. T lymphocytes specific for this neoantigen, generated from healthy donors, were shown to kill CBFB-MYH11 ${ }^{+}$HLA-B * 40:01 ${ }^{+}$AML cell lines and primary human AML samples in vitro inhibited the in vivo growth of AML cells in a patient-derived murine xenograft model. In an applicative perspective, the authors successfully transduced high-avidity $C B F B-M Y H 11$ epitope-specific $\mathrm{T}$ cell receptors into $\mathrm{CD}^{+} \mathrm{T}$ lymphocytes, which acquired antileukemic activity in vitro. In addition to reporting the first neoantigen derived from an AML fusion protein, the study has the merit of emphasizing the concept that targeting neoantigens has clinical relevance even in low-mutational frequency cancers like fusion-driven AML. This work also provides the rationale supporting the development of adoptive immunotherapy for AML, based on the infusion of autologous T lymphocytes engineered to express a T-cell receptor specific for a recurrent fusion-derived neoantigen epitope.

\section{CART Cells: A New Dawn in Cancer Immunotherapy for Diffuse Large B Cell Lymphoma \\ Highlighted by Alessandro Busca}

Over the last 10 to 15 years the treatment of patients with hematologic malignancies has seen the blossoming of a large number of new agents and even new treatment strategies. Among these, chimeric antigen receptor-engineered T (CART) cells, have revolutionized the therapeutic paradigm of patients with B-cell lymphoid malignancies and acute lymphoblastic leukemia (ALL).

CART cells are genetically modified T lymphocytes of the patient which are collected through an unstimulated leukapheresis. Lenti or retroviral vectors are used to introduce into T-lymphocytes a gene encoding the engineered chimeric antigen receptor targeting the antigen CD19.

Currently in Europe, two commercial products are available for the treatment of relapsed or refractory diffuse large B cell lymphoma and primary mediastinal large B-cell lymphoma after two or more lines of treatment, and in patients up to 25 years of age with B-ALL that are refractory or in second or later relapse. A more limited experience has been reported in patients with $\mathrm{R} / \mathrm{R}$ B-ALL with complete remission rates as high as 68-93\%, although relapse remains a major issue occurring in $40-50 \%$ of patients $[31,32]$.

Three pivotal studies showed anti-lymphoma activity with overall response rates ranging from 59\% to $83 \%$ [33-35]. Certainly, longer follow-up is mandatory to define the real relevance of this approach, and all efforts to further implement research in this field should be commended.

17. FDG PET/CT Lesion Dissemination and Metabolic Tumor Burden: Two New, Complementary Risk Factors in Diffuse Large B Cell Lymphoma

Highlighted by Annibale Versari

The spread of lesions is a prognostic factor in DLBCL. The article published by Cottereau et al. confirmed in the 290 DLBCL patients of the REMARC study the prognostic 
impact of a new dissemination index determined in pre-therapeutic PET, the SDmax, the greatest distance existing between tumor lesions [36]. This new biomarker, initially described on a population of patients of the LNH073B trial [37], makes it possible to separate patients from the REMARC trial all good responders to six courses of R-CHOP, patients with high SDmax $\left(>0.32 \mathrm{~m}^{-1}\right)$ whose progression-free survival (PFS) and overall survival (OS) at four years are respectively $46 \%$ and $71 \%$, and low SDmax patients with $77 \%$ and $87 \%$ survival. This biomarker is in this respect superior to IPI and NCCN-IPI. Multivariate analysis showed that SDmax and metabolic volume (TMTV $>220 \mathrm{~cm}^{3}$ ) independently predicted progression-free survival and overall survival and that the combination of the two parameters identified a very high risk group of patients with PFS of $41 \%$ and OS of $66 \%$. SDmax and TMTV are two new, complementary risk factors that may add to existing prognostic models.

18. Can Artificial Intelligence Become the Blue Fairy of Pathologists for the Diagnosis of Myeloproliferative Neoplasms?

Highlighted by Jean-Jacques Kiladjian

Bone marrow histopathology is a key diagnostic feature for myeloproliferative neoplasms (MPN) in the latest World Health Organization 2016 classification [38]. However, since many of the studied parameters in bone marrow biopsies are subjective or semiquantitatively measured, there has been some concern about reliability and reproducibility of MPN diagnosis based on histological interpretation [39]. Sirinukunwattana et al. [40] have recently assessed the role of artificial intelligence (AI) in helping pathologists in their interpretation of bone marrow trephines for suspected MPN. They focused on one of the key and highly subjective parameters: megakaryocytes' cytological and topographic features. They showed that digital images derived from bone marrow sections could be accurately classified, and improved diagnosis using an automated workflow with support of AI models. However, the eye of the pathologist is still required, and we will hopefully still have many occasions to discuss with our colleagues the clinical and molecular features for a solid MPN multidisciplinary diagnosis.

\section{Targeting CD70-Promoted Blast Stemness as a Promising Strategy to Overcome Relapse in HMA-Treated Elderly Patients? \\ Highlighted by Francesco Onida}

Despite limited responses and high relapse rate, hypomethylating agents (HMAs) nowadays represent the prevailing treatment option in elderly patients with AML because of the relatively low toxicity profile. Cell-autonomous CD70/CD27 signaling was previously shown to promote blast stemness in AML [41]. In this paper [42], the same authors prove that HMA treatment induces upregulation of the TNF family ligand CD70 and its receptor CD27 in leukemia stem cells (LSCs). They also elegantly show that combination of a blocking $\alpha$ CD70 monoclonal antibody with HMAs significantly reduces the LCS growth in vitro by blocking the CD70/CD27 interaction. Likewise, $\alpha$ CD70/decitabine treatment in mice is proved to reduce leukemic engraftment in patient-derived xenograft, while the ADCC-enhanced $\alpha \mathrm{CD} 70 \mathrm{mAb}$ cusatuzumab in the presence of NK cells further reduces leukemia engraftment and LSC numbers. In the following phase 1 trial in 12 AML elderly patients treated with a cusatuzumab/5-AZA combination, hematological responses were observed in all of them, including $8 \mathrm{CR}$, together with a substantial reduction of LCSs. Whether deterioration of leukemia blast stemness by cusatuzumab may translate to a longterm reduction of relapse in AML patients treated with HMAs will be possibly unveiled by the ongoing phase 2 trial.

Funding: This research received no external funding.

Conflicts of Interest: The authors declare no conflict of interest. 


\section{References}

1. Boyle, E.M.; Ashby, C.; Tytarenko, R.G.; Deshpande, S.; Wang, H.; Wang, Y.; Rosenthal, A.; Sawyer, J.; Tian, E.; Flynt, E.; et al. BRAF and DIS3 Mutations Associate with Adverse Outcome in a Long-term Follow-up of Patients with Multiple Myeloma. Clin. Cancer Res. 2020, 26, 2422-2432. [CrossRef]

2. Walker, B.A.; Mavrommatis, K.; Wardell, C.P.; Ashby, T.C.; Bauer, M.; Davies, F.E.; Rosenthal, A.; Wang, H.; Qu, P.; Hoering, A.; et al. A high-risk, Double-Hit, group of newly diagnosed myeloma identified by genomic analysis. Leukemia 2019, 33, 159-170. [CrossRef]

3. Liu, H.; He, J.; Koh, S.P.; Zhong, Y.; Liu, Z.; Wang, Z.; Zhang, Y.; Li, Z.; Tam, B.T.; Lin, P.; et al. Reprogrammed marrow adipocytes contribute to myeloma-induced bone disease. Sci. Transl. Med. 2019, 11, eaau9087. [CrossRef] [PubMed]

4. Fairfield, H.; Dudakovic, A.; Khatib, C.M.; Farrell, M.; Costa, S.; Falank, C.; Hinge, M.; Murphy, C.S.; DeMambro, V.; Pettitt, J.A.; et al. Myeloma-modified adipocytes exhibit metabolic dysfunction and a senescence-associated secretory phenotype (SASP). Cancer Res. 2020, 81, 634-647. [CrossRef] [PubMed]

5. Moreau, P.; Chanan-Khan, A.; Roberts, A.W.; Agarwal, A.B.; Facon, T.; Kumar, S.; Touzeau, C.; Punnoose, E.A.; Cordero, J.; Munasinghe, $\mathrm{W}$; ; et al. Promising efficacy and acceptable safety of venetoclax plus bortezomib and dexamethasone in relapsed/refractory MM. Blood 2017, 130, 2392-2400. [CrossRef]

6. Kumar, S.K.; Harrison, S.J.; Cavo, M.; de la Rubia, J.; Popat, R.; Gasparetto, C.; Hungria, V.; Salwender, H.; Suzuki, K.; Kim, I.; et al. Venetoclax or placebo in combination with bortezomib and dexamethasone in patients with relapsed or refractory multiple myeloma (BELLINI): A randomised, double-blind, multicentre, phase 3 trial. Lancet Oncol. 2020, 21, 1630-1642. [CrossRef]

7. Frangoul, H.; Altshuler, D.; Cappellini, M.D.; Chen, Y.-S.; Domm, J.; Eustace, B.K.; Foell, J.; de la Fuente, J.; Grupp, S.; Handgretinger, R.; et al. CRISPR-Cas9 Gene Editing for Sickle Cell Disease and $\beta$-Thalassemia. N. Engl. J. Med. 2021, 384, 252-260. [CrossRef] [PubMed]

8. Frangoul, H.; Bobruff, Y.; Cappellini, M.D.; Corbacioglu, S.; Fernandez, C.M.; de la Fuente, J.; Grupp, S.A.; Handgretinger, R.; Ho, T.W.; Imren, S.; et al. Safety and efficacy of CTX001 in patients with transfusion-dependent $\beta$-thalassemia or sickle cell disease: Early results from the CLIMB THAL-111 and CLIMB SCD-121 studies of autologous CRISPR-CAS9-modified CD34+ hematopoietic stem and progenitor cells. In Proceedings of the 62nd American Society of Hematology Annual Meeting, Online, 5-8 December 2020.

9. DiNardo, C.D.; Pratz, K.; Pullarkat, V.; Jonas, B.A.; Arellano, M.; Becker, P.S.; Frankfurt, O.; Konopleva, M.; Wei, A.H.; Kantarjian, H.M.; et al. Enetoclax combined with decitabine or azacitidine in treatment-naive, elderly patients with acute myeloid leukemia. Blood 2019, 133, 7-17. [CrossRef]

10. DiNardo, C.D.; Jonas, B.A.; Pullarkat, V.; Thirman, M.J.; Garcia, J.S.; Wei, A.H.; Konopleva, M.; Döhner, H.; Letai, A.; Fenaux, P.; et al. Azacitidine and Venetoclax in Previously Untreated Acute Myeloid Leukemia. N. Engl. J. Med. 2020, 383, 617-629. [CrossRef]

11. Röllig, C.; Kramer, M.; Schliemann, C.; Mikesch, J.H.; Steffen, B.; Krämer, A.; Noppeney, R.; Schäfer-Eckart, K.; Krause, S.W.; Hänel, M.; et al. Does time from diagnosis to treatment affect the prognosis of patients with newly diagnosed acute myeloid leukemia? Blood 2020, 136, 823-830. [CrossRef]

12. Sallman, D.A.; Komrokji, R.; Vaupel, C.; Cluzeau, T.; Geyer, S.M.; McGraw, K.L.; Al Ali, N.H.; Lancet, J.; McGinniss, M.J.; Nahas, S.; et al. Impact of TP53 mutation variant allele frequency on phenotype and outcomes in myelodysplastic syndromes. Leukemia 2016, 30, 666-673. [CrossRef] [PubMed]

13. Montalban-Bravo, G.; Kanagal-Shamanna, R.; Benton, C.B.; Class, C.A.; Chien, K.S.; Sasaki, K.; Naqvi, K.; Alvarado, Y.; Kadia, T.M.; Ravandi, F.; et al. Genomic context and TP53 allele frequency define clinical outcomes in TP53-mutated myelodysplastic syndromes. Blood Adv. 2020, 4, 482-495. [CrossRef] [PubMed]

14. Short, N.J.; Montalban-Bravo, G.; Hwang, H.; Ning, J.; Franquiz, M.J.; Kanagal-Shamanna, R.; Patel, K.P.; DiNardo, C.D.; Ravandi, F.; Garcia-Manero, G.; et al. Prognostic and therapeutic impacts of mutant TP53 variant allelic frequency in newly diagnosed acute myeloid leukemia. Blood Adv. 2020, 4, 5681-5689. [CrossRef] [PubMed]

15. Schmitz, N.; Truemper, L.H.; Bouabdallah, K.; Ziepert, M.; Leclerc, M.; Cartron, G.; Jaccard, A.; Reimer, P.; Wagner-Drouet, E.M.; Wilhelm, M.; et al. A randomized phase 3 trial of auto vs. allo transplantation as part of first-line therapy in poor-risk peripheral T-NHL. Blood 2020. [CrossRef]

16. Ono, T.; Mimuro, J.; Madoiwa, S.; Soejima, K.; Kashiwakura, Y.; Ishiwata, A.; Takano, K.; Ohmori, T.; Sakata, Y. Severe secondary deficiency of von Willebrand factor-cleaving protease (ADAMTS13) in patients with sepsis-induced disseminated intravascular coagulation: Its correlation with development of renal failure. Blood 2006, 107, 528-534. [CrossRef]

17. Mancini, I.; Baronciani, L.; Artoni, A.; Colpani, P.; Biganzoli, M.; Cozzi, G.; Novembrino, C.; Boscolo Anzoletti, M.; De Zan, V.; Pagliari, M.T.; et al. The ADAMTS13-von Willebrand factor axis in COVID-19 patients. J. Thromb. Haemost. 2020. [CrossRef]

18. Conway, E.M. Reincarnation of ancient links between coagulation and complement. J. Thrombos. Haemostas. 2015, 13 (Suppl. S1), S121-S132. [CrossRef]

19. Ramlall, V.; Thangaraj, P.M.; Meydan, C.; Foox, J.; Butler, D.; Kim, J.; May, B.; De Freitas, J.K.; Glicksberg, B.S.; Mason, C.E.; et al. Immune complement and coagulation dysfunction in adverse outcomes of SARS-CoV-2 infection. Nat. Med. 2020, 26, 1609-1615. [CrossRef]

20. Mastellos, D.C.; Pires da Silva, B.G.P.; Fonseca, B.A.L.; Fonseca, N.P.; Auxiliadora-Martins, M.; Mastaglio, S.; Ruggeri, A.; Sironi, M.; Radermacher, P.; Chrysanthopoulou, A.; et al. Complement C3 vs. C5 inhibition in severe COVID19: Early clinical findings reveal differential biological efficacy. Clin. Immunol. 2020, 220, 108598. [CrossRef] 
21. Sancho-Albero, M.; Sebastián, V.; Sesé, J.; Pazo-Cid, R.; Mendoza, G.; Arruebo, M.; Martín-Duque, P.; Santamaría, J. Isolation of exosomes from whole blood by a new microfluidic device: Proof of concept application in the diagnosis and monitoring of pancreatic cancer. J. Nanobiotechnol. 2020, 18, 150. [CrossRef]

22. Azizian, A.; Rühlmann, F.; Krause, T.; Bernhardt, M.; Jo, P.; König, A.; Kleiß, M.; Leha, A.; Ghadimi, M.; Gaedcke, J. CA19-9 for detecting recurrence of pancreatic cancer. Sci. Rep. 2020, 10, 1332. [CrossRef]

23. Signore, A.; Lauri, C.; Auletta, S.; Varani, M.; Onofrio, L.; Glaudemans, A.W.J.M.; Panzuto, F.; Marchetti, P. Radiopharmaceuticals for Breast Cancer and Neuroendocrine Tumors: Two Examples of How Tissue Characterization May Influence the Choice of Therapy. Cancers (Basel) 2020, 12, 781. [CrossRef]

24. Krishnan, A.; Adhikarla, V.; Poku, E.K.; Palmer, J.; Chaudhry, A.; Biglang-Awa, V.E.; Bowles, N.; Nathwani, N.; Rosenzweig, M.; Sahebi, F.; et al. Identifying CD38+ cells in patients with multiple myeloma: First-in-human imaging using copper-64-labeled daratumumab. Blood Adv. 2020, 4, 5194-5202. [CrossRef]

25. Reinke, S.; Bröckelmann, P.J.; Iaccarino, I.; Garcia-Marquez, M.; Borchmann, S.; Jochims, F.; Kotrova, M.; Pal, K.; Brüggemann, M.; Hartmann, E.; et al. Tumor and microenvironment response but no cytotoxic T-cell activation in classic Hodgkin lymphoma treated with anti-PD1. Blood 2020, 136, 2851-2863. [CrossRef]

26. Mertoglu, C.; Huyut, M.T.; Arslan, Y.; Ceylan, Y.; Coban, T.A. How do routine laboratory tests change in coronavirus disease 2019? Scand. J. Clin. Lab. Investig. 2020, 81, 24-33. [CrossRef]

27. Ponti, G.; Maccaferri, M.; Ruini, C.; Tomasi, A.; Ozben, T. Biomarkers associated with COVID-19 disease progression. Crit. Rev. Clin. Lab. Sci. 2020, 57, 389-399. [CrossRef] [PubMed]

28. Sun, S.; Cai, X.; Wang, H.; He, G.; Lin, Y.; Lu, B.; Chen, C.; Pan, Y.; Hu, X. Abnormalities of peripheral blood system in patients with COVID-19 in Wenzhou, China. Clin. Chim. Acta 2020, 507, 174-180. [CrossRef] [PubMed]

29. McGranahan, N.; Swanton, C. Neoantigen quality, not quantity. Sci. Transl. Med. 2019, 11, eaax7918. [CrossRef] [PubMed]

30. Biernacki, M.A.; Foster, K.A.; Woodward, K.B.; Coon, M.E.; Cummings, C.; Cunningham, T.M.; Dossa, R.G.; Brault, M.; Stokke, J.; Olsen, T.M.; et al. CBFB-MYH11 fusion neoantigen enables T cell recognition and killing of acute myeloid leukemia. J. Clin. Investig. 2020, 130, 5127-5141. [CrossRef]

31. Maude, S.L.; Laetsch, T.W.; Buechner, J.; Rives, S.; Boyer, M.; Bittencourt, H.; Bader, P.; Verneris, M.R.; Stefanski, H.E.; Myers, G.D.; et al. Tisagenlecleucel in children and young adults with B-cell lymphoblastic leukemia. N. Engl. J. Med. 2018, 378, 439-448. [CrossRef]

32. Park, J.H.; Rivière, I.; Gonen, M.; Wang, X.; Sénéchal, B.; Curran, K.J.; Sauter, C.; Wang, Y.; Santomasso, B.; Mead, E.; et al. Long-term follow-up of CD19 CAR therapy in acute lymphoblastic leukemia. N. Engl. J. Med. 2018, 378, 449-459. [CrossRef]

33. Neelapu, S.S.; Locke, F.L.; Bartlett, N.L.; Lekakis, L.J.; Miklos, D.B.; Jacobson, C.A.; Braunschweig, I.; Oluwole, O.O.; Siddiqi, T.; Lin, Y.; et al. Axicabtagene Ciloleucel CAR T-Cell Therapy in Refractory Large B-Cell Lymphoma. N. Eng. J. Med. 2017, 377, 2531-2544. [CrossRef]

34. Schuster, S.S.; Bishop, M.R.; Tam, C.S.; Waller, E.K.; Borchmann, P.; McGuirk, J.P.; Jäger, U.; Jaglowski, S.; Andreadis, C.; Westin, J.R.; et al. Tisagenlecleucel in Adult Relapsed or Refractory Diffuse Large B-Cell Lymphoma. N. Eng. J. Med. 2019, 380, 45-56. [CrossRef] [PubMed]

35. Abramson, J.S.; Palomba, M.L.; I Gordon, L.; A Lunning, M.; Wang, M.; Arnason, J.; Mehta, A.; Purev, E.; Maloney, D.G.; Andreadis, C.; et al. Lisocabtagene maraleucel for patients with relapsed or refractory large B-cell lymphomas (TRANSCEND NHL 001): A multicentre seamless design study. Lancet 2020, 396, 839-852. [CrossRef]

36. Cottereau, A.-S.; Meignan, M.; Nioche, C.; Capobianco, N.; Clerc, J.; Chartier, L.; Vercellino, L.; Casasnovas, O.; Thieblemont, C.; Buvat, I. Risk stratification in diffuse large B-cell lymphoma using lesion dissemination and metabolic tumor burden calculated from baseline PET/CT. Ann. Oncol. 2020, in press. [CrossRef] [PubMed]

37. Cottereau, A.-S.; Nioche, C.; Dirand, A.-S.; Clerc, J.; Morschhauser, F.; Casasnovas, O.; Meignan, M.A.; Buvat, I. 18F-FDG PET Dissemination Features in Diffuse Large B-Cell Lymphoma Are Predictive of Outcome. J. Nucl. Med. 2019, 61, 40-45. [CrossRef]

38. Arber, D.A.; Orazi, A.; Hasserjian, R.; Thiele, J.; Borowitz, M.J.; Le Beau, M.M.; Bloomfield, C.D.; Cazzola, M.; Vardiman, J.W. The 2016 revision to the World Health Organization classification of myeloid neoplasms and acute leukemia. Blood 2016, 127, 2391-2405. [CrossRef] [PubMed]

39. Nand, S.; Spivak, J.L.; Barbui, T.; Thiele, J.; Tefferi, A. Myeloproliferative Neoplasms. N. Engl. J. Med. 2017, 377, 894-896. [CrossRef]

40. Sirinukunwattana, K.; Aberdeen, A.; Theissen, H.; Sousos, N.; Psaila, B.; Mead, A.J.; Turner, G.D.H.; Rees, G.; Rittscher, J.; Royston, D. Artificial intelligence-based morphological fingerprinting of megakaryocytes: A new tool for assessing disease in MPN patients. Blood Adv. 2020, 4, 3284-3294. [CrossRef]

41. Riether, C.; Schürch, C.M.; Bührer, E.D.; Hinterbrandner, M.; Huguenin, A.-L.; Hoepner, S.; Zlobec, I.; Pabst, T.; Radpour, R.; Ochsenbein, A.F. CD70/CD27 signaling promotes blast stemness and is a viable therapeutic target in acute myeloid leukemia. J. Exp. Med. 2017, 214, 359-380. [CrossRef] [PubMed]

42. Riether, C.; Pabst, T.; Höpner, S.; Bacher, U.; Hinterbrandner, M.; Banz, Y.; Müller, R.; Manz, M.G.; Gharib, W.H.; Francisco, D.; et al. Targeting CD70 with cusatuzumab eliminates acute myeloid leukemia stem cells in patients treated with hypomethylating agents. Nat. Med. 2020, 26, 1459-1467. [CrossRef] [PubMed] 